\title{
Sleep assessment in individuals with cancer
}

\section{Keywords: sleep disorders, cancer, depression, anxiety, insomnia}

Abbreviations: INCA, the national cancer institute José Alencar Gomes da Silva; ICSD-3, third international classification of sleep disorders; AASM, American association sleep medicine; ICU, intensive care unit; OSA, obstructive sleep apnea; IH, intermittent hypoxemia; EDS, excessive daytime sleepiness

\section{Introduction}

Sleep disorders are increasingly recognized as an important cause of morbidity and mortality. They are among the most common complications that individuals diagnosed with cancer live with during treatment and when untreated have a substantial economic and social impact. Currently, subjective instruments are used to assess sleep patterns in cancer patients, since there is little information investigating sleep disorders in these individuals. Recognizing these changes in sleep patterns is essential as symptoms can interfere with quality of life and well-being.

\section{Sleep and cancer}

Sleep is defined as a restorative and healthy stage, regulated by endogenous and exogenous factors that coincide over a 24-hour period, being called the circadian cycle or biological clock. Any change in lifestyle can lead to changes in this cycle and, consequently, in sleep. The National Cancer Institute José Alencar Gomes da Silva (INCA) describes cancer as a disorderly growth of cells that invade tissues and organs. These cells tend to be very aggressive and uncontrollable, being able to generate tumors and spread to other regions of the body. ${ }^{2}$ According to INCA, an estimated 625 thousand new cases of cancer in Brazil, in the years 2020/2022. The division of incidence by geographic region shows that the Southeast region concentrates more than $60 \%$ of the incidence, with a predominance of prostate and female breast cancers, as well as lung and intestine. ${ }^{2}$ Cancer is one of the main causes of illness and death in the world, affecting millions of people, partly due to population growth, aging, as well as changes in the distribution and prevalence of cancer risk factors, especially those associated with socioeconomic development. ${ }^{3,4}$ In patients diagnosed with cancer, the sleep regulatory mechanism is altered, being interrupted for a variety of reasons, such as difficulty in initiating sleep, the presence of nightmares, daytime sleepiness, insomnia, waking up in the middle of the night, staying awake for a long period, difficulty falling asleep and waking up very early. ${ }^{3}$

In 2014, the third international classification of sleep disorders (ICSD-3) was published by the American Association Sleep Medicine (AASM) detailing sleep disorders in 7 categories: Insomnia; Respiratory disorders related to sleep; Central disorders of hypersomnia; Sleep-wake disorders of circadian rhythm; Parasomnias; Movement disorders related to sleep and other sleep disorders. ${ }^{5}$ These sleep disorders are more frequent in cancer patients when related to the general population. The prevalence of these sleep disorders in patients varies from $24 \%$ to $95 \%$ and the literature showed an association between prognosis, clinical evolution of cancer and poor sleep quality. ${ }^{6,7}$ The association between breast cancer symptoms and poor sleep quality is present in $65 \%$ of women. ${ }^{8,9}$ Sleep disorders in cancer patients can occur at different times in the course
Volume 4 Issue 2 - 2020

\author{
Luísa Teixeira Pasqualotto,' Walquíria da \\ Mata Santos, ${ }^{2}$ Newton Santos Faria Júnior ${ }^{3}$ \\ 'Postgraduate Program in Rehabilitation Sciences Federal \\ University of Alfenas-MG, Brazil \\ ${ }^{2}$ Postgraduate studies in Adult Intensive Care InterFisio BH, \\ Brazil \\ ${ }^{3}$ University of the State of Minas Gerais-Divinópolis Unit, Brazil
}

Correspondence: Newton Santos Faria Júnior, PT, PhD, University of the State of Minas Gerais- UEMG-Divinópolis Unit Av Paraná, 300I, Jardim Belvedere, 35.50I-170, Divinópolis, Minas Gerais, Brazil, Tel (37) 3229-3590,

Email nsdfj@yahoo.com.br

Received: August 05, 2020 | Published: September I5, 2020

of the disease (diagnosis, treatment and terminal phase), resulting in decreased quality of life, impaired mood and reduced energy, and may persist for up to several months or even years after treatment, becoming chronic. ${ }^{10}$ In addition, during cancer treatment, many patients require care in intensive care units (ICUs), which can cause additional sleep disruption. This interruption of sleep in the ICU may be due to environmental factors, frequent interventions and the use of various sedative medications. ${ }^{11}$ Therefore, cancer patients, especially those who need ICU care, commonly develop sleep problems. And the treatment of these problems can improve morbidity and possibly mortality. ${ }^{11}$

\section{Sleep disorders in cancer patients}

In recent years, some studies have demonstrated the possibility of sleep duration and circadian disorders being linked to an increased risk of developing or promoting adverse cancer outcomes. ${ }^{12}$ Among sleep disorders, obstructive sleep apnea (OSA), a clinical condition characterized by recurrent episodes of partial or complete upper airway obstruction during sleep, which can generate intermittent hypoxemia (IH) and sleep fragmentation, can contribute to altered cancer results and tumor growth. ${ }^{12,13}$ It is worth mentioning that OSA is a chronic, progressive, disabling disease, with high mortality and morbidity. ${ }^{14}$ Due to IH caused by OSA, there is an increased likelihood of mutation and, therefore, malignant transformation of cells, as well as tumor growth and metastasis. ${ }^{12}$ In relation to other sleep disorders, excessive daytime sleepiness (EDS) characterized by the subjective feeling of need for sleep during the day can be one of the important causes of significant changes in work performance, social interaction and quality of life. ${ }^{15}$ The main factors that contribute to EDS are: sleep quality, time to wake up; associated medical conditions or a general medical condition that could impact sleep. Cancer being considered one of the common medical conditions for the development of EDS. ${ }^{16}$ Another common disorder in these cancer patients is insomnia, which stands out when associated with cancer patients, as many of them report having difficulties sleeping and maintaining sleep, especially during the diagnostic phase (six months pre-diagnosis to eighteen months after diagnosis). ${ }^{17}$ Insomnia commonly associated with 
stressful events, such as clinical symptoms of the disease, treatment, side effects, palliative care and terminal stages, can also be linked to tumor invasion, which for this reason the patient presents with pain and discomfort leading to difficulty in start sleep. ${ }^{17}$ These symptoms negatively affect the quality of life and can aggravate the levels of anxiety and depression faced by these patients during the treatment of the disease. It is not yet clear whether these sleep disorders can lead to cancer progression or development, leaving gaps for further studies. ${ }^{12}$

\section{Quality of life}

According to the World Health Organization, quality of life is defined as the individual's perception of cultural, social, political and economic influences, in order to achieve their goals and expectations, which represents well-being with their life. It involves emotional, physical, mental and psychological satisfaction, as well as family and social relationships. ${ }^{18}$ Recognizing changes in sleep in cancer patients is essential since their symptoms can negatively impact quality of life and their daytime performance. These changes may be related to adverse effects of treatments, which causes discomfort to the patient and a negative impact on their quality of life. ${ }^{19}$ The quality of life in these individuals has been extensively studied during these years, considering this impact and its vulnerability. ${ }^{20}$ Sleep disorders are associated with health conditions and, consequently, with quality of life. ${ }^{21}$ Being able to increase the chances of emotional disorders, decrease daily productivity, aggravate health problems, among other important commitments. ${ }^{22}$ In this context, the role of the health team in the care of these patients during and after cancer treatment is important so that they can contribute to the well-being and quality of life of these individuals.

\section{Anxiety and depression}

The difficulty in having a good quality of sleep in individuals with cancer can be associated, in addition to the diagnosis, with anxiety and depression, which may occur during or after treatment. ${ }^{23}$ In these patients, the probability of developing anxiety, depression, low selfesteem, stress and sleep-related problems is high, despite the fact that cancer treatment has currently achieved a high level of success. ${ }^{24,25}$ The symptoms related to treatment, such as fatigue, nausea, pain and discomfort, lead to these conditions. ${ }^{23}$ The family also goes through this anxiety and stress, a common factor, due to the shock and acceptance of reality. However, these have a fundamental role in the improvement or worsening of cancer patients, and may provide support or even lead to abandonment caused by the impossibility of help, which can mitigate the depression of this patient. ${ }^{26}$ The patient diagnosed with cancer is fragile and when he does not have the support and help of his family makes the healing process even more difficult. The help of this family member can help in understanding this complex moment, in addition to performing tasks that may be difficult during this period. It is believed that this relationship of family support can minimize sadness, stress, fear and anxiety. ${ }^{23}$ Insomnia affects up to $50 \%$ of individuals with cancer, being able to lead to fatigue, mood disorders, contributing to immunosuppression, which can have a profound impact on the lives of these patients. ${ }^{27,28}$ Sleep disorders, depression and hopelessness are common symptoms and are present before the start of chemotherapy and can last after clinical treatment, negatively affecting quality of life. ${ }^{29,30}$

\section{Sleep hygiene in cancer}

Sleep hygiene measures, despite being relevant for the entire population, are of special interest to patients with different pathologies, standing out in cancer patients, with the general objective of facilitating restful and sufficient sleep. ${ }^{31}$ Adherence to sleep hygiene components, had a relative increase over time between $68 \%$ and $78 \%$ compared to the other components of several treatments used by patients. In addition to good sleep hygiene, it is recommended to avoid periods of sleep deprivation..$^{32}$ Although non-pharmacological therapy is a safe and effective way to treat sleep disorders, many of its components can be difficult to apply to patients undergoing cancer treatment. ${ }^{17}$ Individuals diagnosed with cancer who are hospitalized can spend many hours of their day in bed, which makes it difficult to implement techniques such as stimulus control, hygiene and sleep restriction. ${ }^{17}$ The hospital environment is often not conducive to a good night's sleep, due to poor lighting, noise and frequent interruptions to procedures and continuous monitoring. And the effects related to cancer treatment, such as fatigue and nausea, can make it difficult to sleep also during the day. ${ }^{17}$ Therefore, it is necessary to include therapeutic and rehabilitation measures that aim to mitigate these sleep changes based on the history, condition and place that this patient is, whether in hospitals or at home.

\section{Conclusion}

The management of these sleep disorders begins with a good and careful assessment to identify the factors and causes. After this assessment, a care plan can be created to provide good sleep quality, identifying medication schemes that promote sleep and reduce fatigue or suggesting non-pharmacological interventions based on the patient's individual needs and desires as previously mentioned. Identifying sleep disorders in these cancer patients is essential, given that poor sleep quality is among the most frequent problems with which these individuals live during treatment. When these sleep disorders are not recognized and treated, a substantial economic and social impact can occur, which can lead to depression, anxiety and repercussions on quality of life. In addition, all members of the healthcare team need to be aware of the intervention to implement changes and alternatives to meet patients' sleep and energy needs.

\section{Acknowledgments}

None.

\section{Conflicts of interest}

The authors declare that they have no conflicts of interests.

\section{Funding}

None.

\section{References}

1. Nascimento JV. Sleep disorders in ICU patients: an integrative review. course conclusion paper (graduation in nursing)-Paraíba state university, Campina Grande, 2016.

2. Inca. Estimate 2019: Incidence of cancer in Brazil/National cancer institute josé alencar gomes da silva. coordination of prevention and surveillance. Rio de Janeiro: Inca, 2019.

3. Kaplow R. Sleep deprivation and psychosocial impact in acutely ill cancer patients. Crit Care Nurs Clin North Am. 2005;17(3):225-237.

4. Bray F, Ferlay J, Soerjomataram I, et al.Global cancer statistics 2018: globocan estimates of incidence and mortality worldwide for 36 cancers in 185 countries. CA Cancer J Clin. 2018;68(6):394-424.

5. Sateia MJ. International classification of sleep disorders-third edition: highlights and modifications. Chest. 2014;146(5):1387-1394. 
6. Palesh OG, Roscoe JA, Mustian KM, et al. Prevalence demographics and psychological associations of sleep disruption in patients with cancer: university of Rochester cancer center community clinical oncology program. J Clin Oncol. 2010;28(2):292-298.

7. Nishura M, Tamura A, Nagai H, et al. Assessment of sleep disturbance in lung cancer patients: relationship between sleep disturbance and pain, fatigue, quality of life, and psychological distress. Palliat Support Care. 2015;13(3):575-581.

8. Van OC, Paul SM, Lee K, et al. Trajectories of sleep disturbance and daytime sleepiness in women before and after surgery for breast cancer. $J$ Pain Symptom Manage. 2013;45(2):244-260.

9. Adams RJ, Appleton SL, Taylor AW, et al.Sleep health of Australian adults in results of the sleep health foundation national survey. Sleep Health. 2017;3(1):35-42.

10. Fabi A, Falcicchio C, Giannarelli D, et al. The course of cancer related fatigue up to ten years in early breast cancer patients: what impact in clinical practice. Breast. 2017;34:44-52.

11. Matthews EE. Sleep disturbances and fatigue in critically ill patients AACN Adv Crit Care.2011;22(3):204-224.

12. Gozal D, Farré R, Nieto FJ. Obstructive sleep apnea and cancer: epidemiologic links and theoretical biological constructs. Sleep Med Rev. 2016;27:43-55

13. Carneiro G, Filho FFR, Togeiro SM, et al. Interactions between obstructive sleep apnea syndrome and insulin resistance. Arq Bras Endocrinol Metabol. 2007;51(7):1035-1040.

14. Mancini MC, Halpern A. Pharmacological treatment of obesity. Arq Bras Endocrinol Metabol. 2002;46(5):497-512.

15. Pereira EF, Teixeira CS, Louzada FM. Daytime sleepiness in adolescents: prevalence and associated factors. Rev Paul Pediatr. 2010;28(1):98-103.

16. Giorelli AS, dos Santos PP, Carnaval T, et al. Excessive daytime sleepiness: clinical, diagnostic and therapeutic aspects. Rev Bras Neurol. 2012;48(3):17-24.

17. Rafihi-Ferreira R, Soares MRZ. Insomnia in patients with breast cancer. Estud Psicol. 2012;29(4):597-607.

18. Whoqol group. The development of the world health organization quality of life assessment instrument (the WHOQOL). In: quality of life assessment: international perspectives. Springer, Berlin, Heidelberg, 1994. 41-57 pp.

19. Freire MEM, Costa SFG, Lima RAG, et al. Health-related quality of life of patients with cancer in palliative care. Texto Contexto Enferm. 2018;27(2):1-13
20. Lopes JV, Bergerot CD, Barbosa LR, et al. Impact of breast cancer and quality of life of women survivors. Rev Bras Enferm. 2018;71(6):29162921.

21. Ropke LM, Souza AG, Bertoz APM, et al. Effect of physical activity on sleep quality and quality of life: a systematized review. Arch Health Invest. 2017;6(12):561-566.

22. Amaro JMRS, Dumith SC. Excessive daytime sleepiness and quality of life related to the health of university professors. J Bras Psiquiatr. 2018;67(2):94-100

23. Silva PR, Cruz LAP, Nascimento TG, et al. Sleep quality and fatigue in women with breast cancer during chemotherapy. Rev Enferm UFSM. 2019;9:e20.

24. Bower JE. Behavioral symptoms in patients with breast cancer and survivors. J Clin Oncol. 2008;26(5):768-777.

25. Reich M, Lesur A, Perdrizet-Chevallier C. Depression, quality of life and breast cancer: a review of the literature. Breast Cancer Res Treat. 2008;110(1):9-17.

26. Vasconcelos AS, Costa C, Barbosa LNF. From anxiety disorder to cancer Rev SBPH. 2008;11(2):51-71.

27. Parker KP, Bliwise DL, Ribeiro M. et al. Sleep/Wake patterns of individuals with advanced câncer measured by ambulatory polysomnography. J Clin Oncol. 2008;26(15):2464-2472.

28. Campos MPO, Hassan BJ, Riechelmann R, et al. Cancer-related fatigue: a review. Rev Assoc Med Bras. 2011;57(2):211-219.

29. Sanford SD, Wagner LI, Beaumont JL, et al. Longitudinal prospective assessment of sleep quality: before, during, and after adjuvant chemotherapy for breast cancer. Support Care Cancer. 2013;21(4):959967.

30. Chen ML, Liu LN, Miaskowski C, et al. Presurgical symptom profiles predict quality of life 2 years after surgery in women with breast cancer. Support Care Cancer. 2016;24(1):243-251.

31. Bahia MG, Soares V, Winck JC. Impact of sleep hygiene on patients with obstructive sleep apnoea syndrome. Rev Port Pneumol. 2006;12(2):147176.

32. Berger AM, Von Essen S, Khun BR, et al. Feasibilty of a sleep intervention during adjuvant breast cancer chemotherapy. Oncol Nurs Forum. 2002;29(10):1431-1441. 